\title{
Launching of ships from horizontal berth by tipping tables - CFD simulation of wave generation
}

\author{
Ivo Senjanovića ${ }^{\text {, Josip Katavića }}{ }^{\text {, Vuko Vukčevića }}{ }^{\text {, Nikola Vladimira }}{ }^{\text {, Hrvoje }}$ \\ Jasak $^{\mathrm{a}, \mathrm{b}}$ \\ ${ }^{a}$ University of Zagreb, Faculty of Mechanical Engineering and Naval Architecture, Ivana \\ Lučića 5, Zagreb, Croatia \\ ${ }^{b}$ Wikki Ltd, 459 Southbank House, SE1 7SJ London, United Kingdom, \\ h.jasak@wikki.co.uk
}

\begin{abstract}
The paper considers the side launching of ships from a horizontal berth by tipping tables. The launching process is split into the six phases and a system of nonlinear differential equations of motion is established. The system is solved using the finite difference method. The numerical calculations of the side launching of a large tanker are performed and the results are compared against the model test results. The generation of a high surface wave is simulated with CFD as a 2D problem. The numerical simulation captures the physics of the side launching with high accuracy. The advantages of building the floating structures on a horizontal berth are pointed out.
\end{abstract}

Keywords: launching, horizontal berth, tipping table, numerical simulation, model test, CFD

\section{Introduction}

Launching of ships and floating structures by tipping table principle is an idea which makes their building on a horizontal berth possible. This enables an improvement of the production process by introducing a high degree of mechanisation and automation, successive block manufacturing and assembling of structural elements, and fitting-out of structures on the way from the workshop to the quay $[1,2,3]$.

The launching system consists of a set of concrete circular slipways builtin into the quay and of the steel turning pads, Fig. 1. The sliding surfaces of the pads are extended to the sliding lines of the building berth. The 


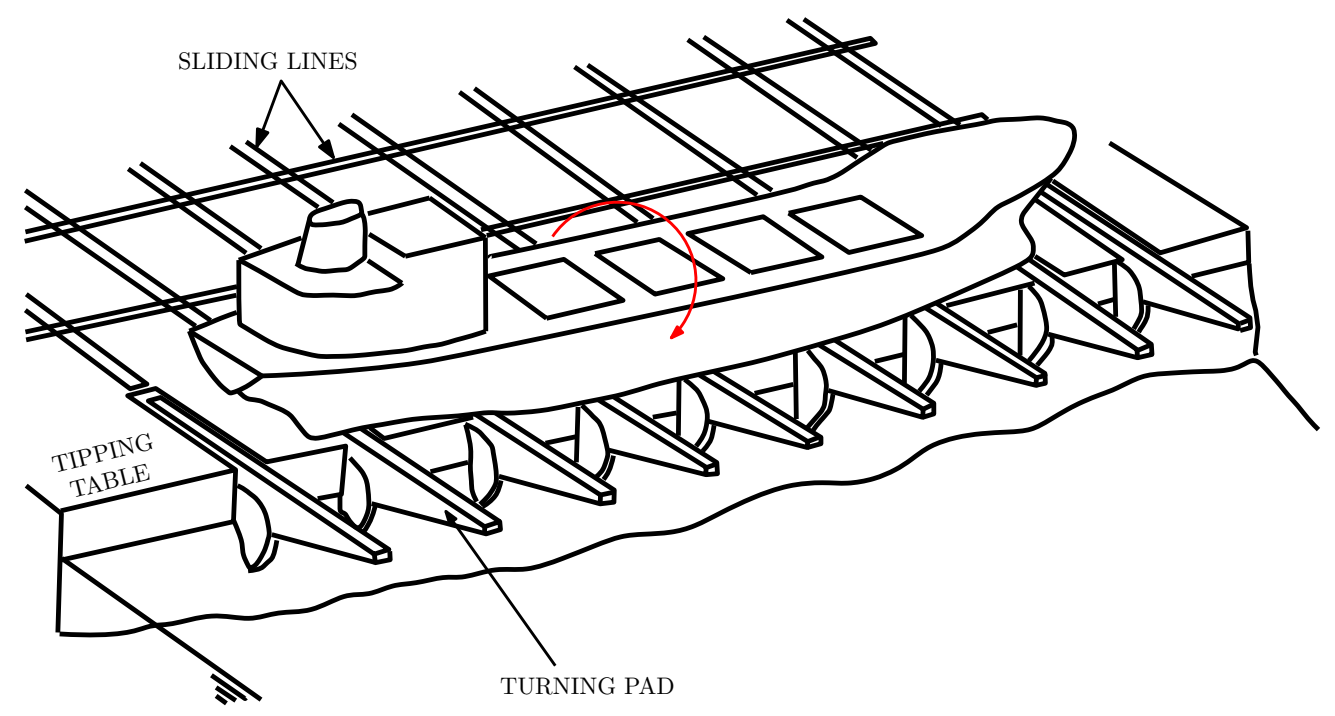

Figure 1: The launching system.

sliding lines and arches are covered by the polymer coating in order to reduce friction $[4]$.

A structure assembled on the packing is pushed onto the braked tipping table by hydraulic gripper jacks. When the moment of structure weight overcomes the friction moment of the sliding arches, the tension forces remove the triggers. The structure then starts to rotate with pads until the displacement excites first its slide along the pads and then its erection in cradles, and finally the floating phase. Thus, the launching system has three degrees of freedom, i.e. pad rotation, $\varphi$, structure rotation, $\vartheta$, and relative structure slide, $s$, Fig. 2. The combinations of the displacements in the air and in the water define different launching phases which are shown in Fig. 3, [5, 6].

Depending on the main characteristics of the launching system it is possible to launch different structures regarding their shape and size, as well as structure blocks, such as grips, docks, platforms etc.; smaller structures being launched longitudinally and larger structures launched sideways. For the longitudinal launching of ships a single pad is sufficient, while for the launching of semi-submersible platforms two pads are necessary. Platforms may be launched sideways, too.

In order to determine the optimal values of the basic parameters of the launching system, an extensive theoretical and experimental investigation has been undertaken. This includes the launching theory, model testing and 


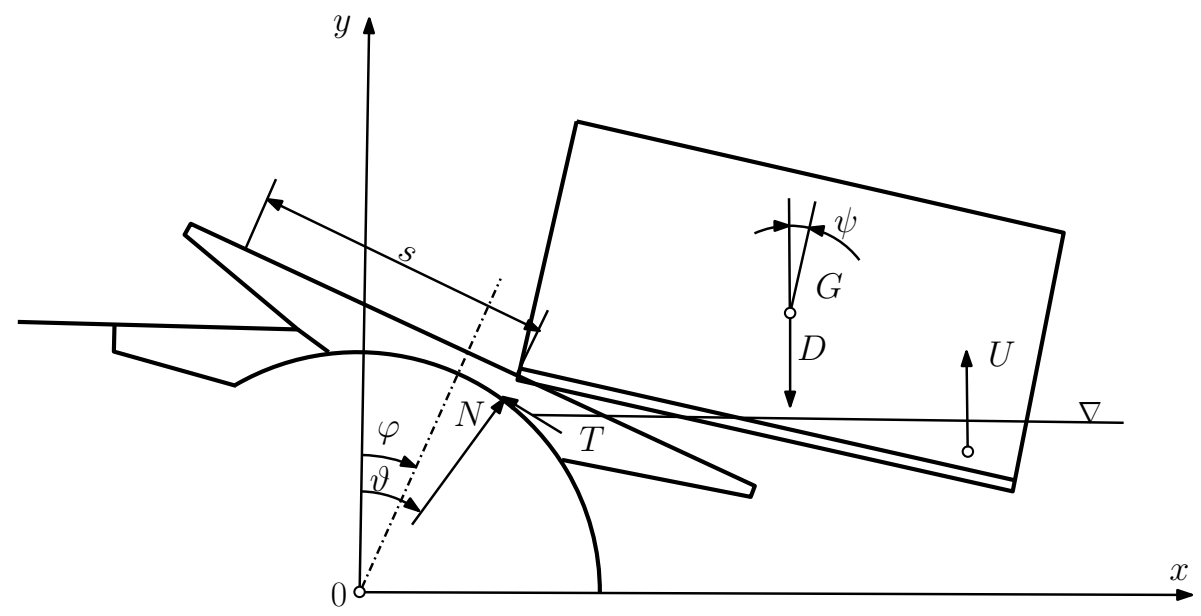

Figure 2: Displacements of the ship and the pad.

structural analysis of the turning pads and launching structures, [5].

The launching theory comprises the definition of the forces acting on the dynamic system of the structure and the pad in each launching phase, i.e. structural weight and inertia, buoyancy, resistance and water inertia, friction forces and slipway reaction; the establishment of equilibrium equations of forces and moments, the derivation of the motion equations by reducing the number of setting equations, specifying the transition conditions from one to another phase, and solving nonlinear differential equations of motion by the finite difference method, [7, 8]. The same advanced solution is achieved by transforming the system of nonlinear differential equations of the second order into extended system of equations of the first order. Then the RungeKutta method is applied, [9].

Special attention is paid to the returning motion of the pad, which is controlled by reducing the buoyancy of the pad, [10].

The procedure for the calculation of longitudinal and side launching of ships and floating structures was computer programmed. The nonlinear differential equations of motion are solved by the finite difference method utilizing an iterative algorithm. A large number of numerical simulations has been performed for launching of pontoons, ships and a semisubmersible platforms, [5, 8].

In order to validate the developed launching theory and the computer program, model tests have been performed in Brodarski Institute - Marine Research 8 Special Technologies in Zagreb. A model of the launching system, 
consisted of only two slipways, was built in a 1:75 scale. A TV camera and a coordinate net were used to record the motions of the launched models. The side launching of three pontoons and two ships was tested as well as the longitudinal launching of a semisubmersible platform, [5].

The side launching of large ships with the trim angle is questionable, since at erection, the ship is supported only by two cradles due to yaw. As a result, a high stress concentration in the ship structure occurs. Furthermore, it is necessary to check the depth of the water and the height of the generated waves.

To investigate the above facts, the model tests of the side launching of large tanker were performed in the Maritime Research Institute Netherlands (MARIN). An existing wooden model in scale 1:68 was adapted for that purpose, $[11,12]$.

In addition, the correlation analysis was performed between the calculated and the measured results, which encouraged the building of the launching system in Shipyard Brodosplit. Five out of the total 13 designed circular slipways of $22 \mathrm{~m}$ were completed. In 1993 the first structure, a 4450 DWT barge, was built on the horizontal berth and side launched by two turning pads, $[13,14]$.

In this paper an illustrative example related to the side launching of a chemical tanker is presented. A correlation analysis of the calculated and measured results is summarised, $[6,12]$. A special attention is paid to the generation of a surface wave during the side launching as a criterion for the application of the current launching system in a shipyard with a limited water basin due to a possible ashore washing. Wave generation is simulated by direct CFD simulation as a 2D problem. 

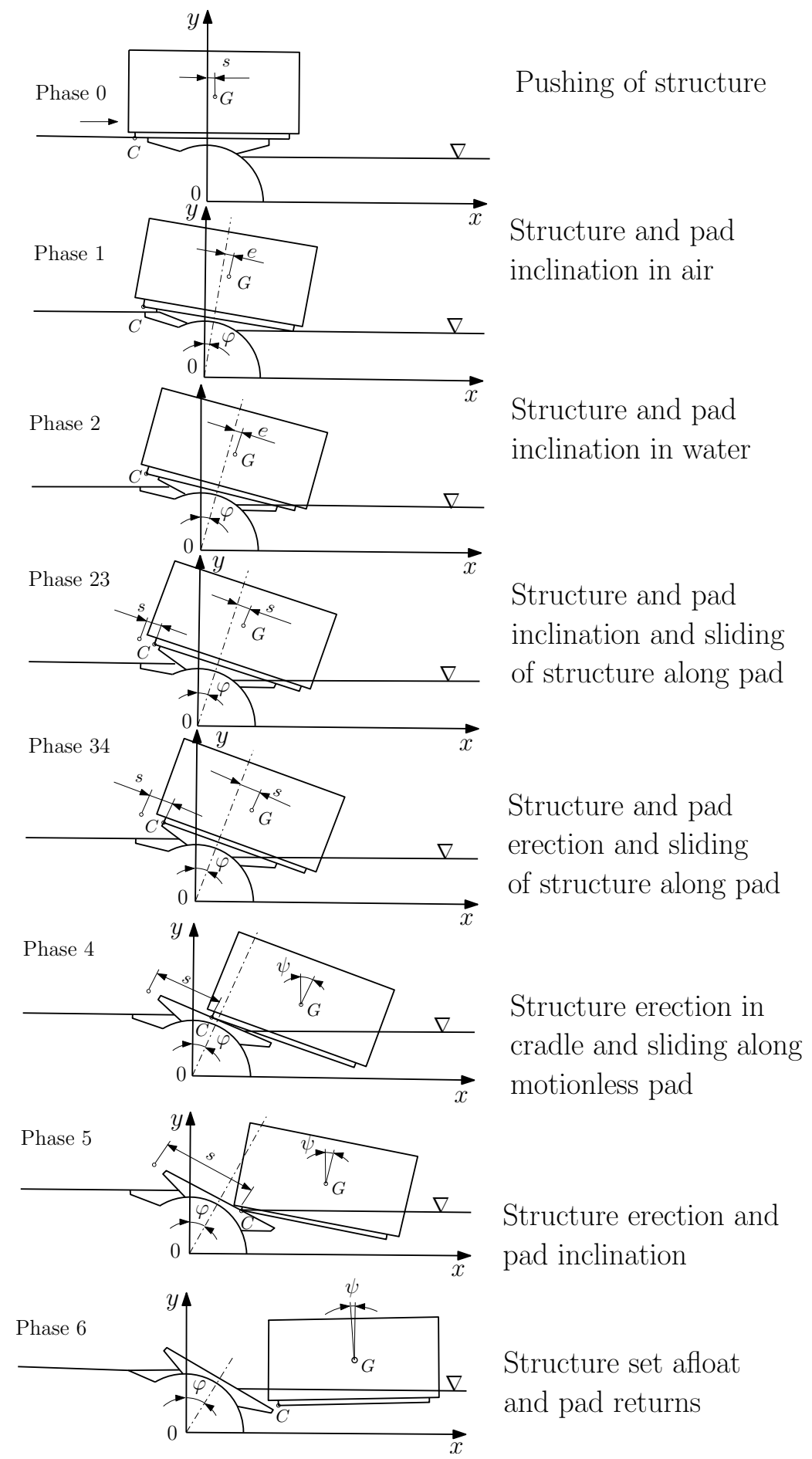

Figure 3: Launching phases. 


\section{Two-phase flow model for the CFD analysis}

This section is dealing with the mathematical model for incompressible, viscous, two-phase flow, which is the basis for the CFD framework. The detailed model can be found in [15], while a brief summary is presented here.

\subsection{Incompressible two-phase flow model}

As both fluids are assumed incompressible, the continuity constraint reads

$$
\nabla \bullet \mathbf{u}=0,
$$

where $\mathbf{u}$ is the velocity field. The momentum equation has the following form

$$
\frac{\partial \mathbf{u}}{\partial t}+\nabla \bullet(\mathbf{u u})-\nabla \bullet\left(\nu_{e} \nabla \mathbf{u}\right)=-\partial 1 \rho \nabla p_{d},
$$

where the decomposition of pressure into the hydrostatic and the dynamic component is used $(p d=p+\rho \mathbf{g} \bullet \mathbf{x}) . \quad \rho$ is the piece-wise constant density field, while $\nu_{e}$ is the effective kinematic viscosity.

The interface between the two fluids is considered infinitesimally thin and the jump interface discontinuities are treated with the Ghost Fluid Method (GFM). In each fluid, density is assumed constant, i.e. $\rho=\rho_{w}$ in water and $\rho=\rho_{a}$ in air. Following the notation used by the GFM authors $[16,17]$, the jump of density across the free surface can be written as

$$
[\rho]=\rho_{w}-\rho_{a},
$$

To facilitate the implementation of the GFM in arbitrary polyhedral finite volume framework, effective kinematic viscosity is assumed continuous across the free surface using the volume fraction $\alpha$ from the VOF approach:

$$
\nu_{e}=\alpha \nu_{e, w}+(1-\alpha) \nu_{e, a},
$$

where $\nu_{e, w}$ is the effective kinematic viscosity in water and $\nu_{e, a}$ is the effective kinematic viscosity in air, allowing the use of general eddy-viscosity turbulence models, [18]. Eq.(4) states that the effective kinematic viscosity does not have a discontinuity across the free surface, although it might have a steep gradient if the smearing of the interface in the VOF approach is confined to a narrow region. 
The kinematic boundary condition $[19,20]$ defines a continuous velocity field across the free surface

$$
[\mathbf{u}]=\mathbf{u}_{w}+\mathbf{u}_{a}=0 .
$$

Eq. (5) states that the velocity field infinitesimally close to the free surface in the water must be the same as the velocity field infinitesimally close to the free surface in the air.

Furthermore, neglecting the surface tension effects as in $[19,20]$ yields a continuous pressure field

$$
[p]=0 .
$$

Taking into account Eqs. (3), (5) and (6), it is possible to arrive at two jump conditions for the dynamic pressure [15]

$$
\begin{gathered}
{\left[p_{d}\right]=-[\rho] \mathbf{g} \bullet \mathbf{x},} \\
{\left[\frac{1}{\rho} \nabla p_{d}\right]=0 .}
\end{gathered}
$$

The jump conditions presented in Eqs. (6),(7) require a representation of the interface between the two fluids. In present work, the capturing of the free surface between is achieved with the VOF method [21]. The VOF method is based on the indicator function $\alpha$, which represents the volume fraction

$$
\alpha=\frac{V_{w}}{V},
$$

where $V_{w}$ is the volume of water inside a control volume $V$. VOF method is conservative because $\alpha$ represents a physical, conserved property, bounded between 0 and 1. Following Rusche [22], transport equation for $\alpha$ reads:

$$
\frac{\partial \alpha}{\partial t}+\nabla \bullet(\mathbf{u} \alpha)+\nabla \bullet\left(\mathbf{u}_{r} \alpha(1-\alpha)\right)=0,
$$

where the last term serves to prevent excessive smearing of the free surface based on the compressive velocity field $u_{r}$ [22]. The term is active only in the smeared interface region due to the $\alpha(1-\alpha)$ non-linear pre-factor.

After the $\alpha$ is advected, the location of the interface given by $\alpha=0.5$ is used in the discretisation of the momentum equation, Eq. (2), obeying the dynamic pressure jump conditions given by Eqs. (7) and (8). 


\section{Overset mesh approach}

The Overset mesh approach refers to the use of multiple disconnected meshes to discretise the flow domain. The component meshes, which can be any size, type, or shape, need only overlap each other to cover the solution domain completely [23]. Furthermore, a component mesh resolving one geometric feature may intersect another geometric feature [24].

This approach has much of its advantages in the computation of multibody and moving body problems as well as in the optimisation studies [24].

Candidate hydrodynamic applications include ships or submarines with rotating propellers, the launch of torpedo or mini-submarines from a parent ship, moving appendages and other control surfaces, ship motion relative to the sea surface and coastline, and seakeeping simulations with multiple ships in close proximity [25].

Overset mesh generation is conceptually split into the off-body or background meshes and a number of near-body meshes which resolve the geometry and the boundary layers. Structured hexahedral meshes are often used for their accuracy. However, the overset technique is routinely applied using the hybrid unstructured meshes for the highly automated meshing of complex configurations [23].

A CFD solution on the system of meshes requires the coupling of the solution between meshes in the overlapped regions. This is typically performed by identifying appropriate inter-mesh boundary locations in one mesh and obtaining the value to be applied by interpolating the solution from meshes that overlap the region [25].

The Domain Connectivity Information (DCI) consists of the locations that are to be excluded from the computation, the location of the intermesh boundary locations, and the corresponding interpolation sources. This domain connectivity information is computed by a code typically called an overset fringe assembly code [25]. The overset fringe assembly algorithm used in this work is described in Subsection 3.2.

\subsection{Overset mesh cell types}

According to their role in the solution process of the governing equations, there are three base overset mesh cell types (also called overset types): acceptor, live and hole cells.

Basic and special overset types are presented in the form of a diagram in Fig. 4. Arrow points from derived to a base type. The diagram should be 
read from the bottom to the top. For example, both master and extended donor are donors, while the donor is a live cell and the live cell is an overset mesh cell.

The live cells are used to discretise the governing equations. Field values in those cells are obtained by solving a system of linear equations.

A donor cell is a special type of the live cell. It is used for overset interpolation, i.e. donor is the interpolation source for providing the value applied to the appropriate acceptor cell.

Field values at acceptor cells are obtained exclusively by interpolation from the donor cells. The field value at an acceptor cell is therefore not affected by the values of its neighbouring cells, but field values at acceptor's neighbouring cells are affected by the field value in the acceptor cell. After the acceptor candidates are identified, the donor search procedure is employed to find the appropriate master donor and extended donors for all acceptors.

The extended donors are neighbours of the master donor, and together with the acceptor and the master donor, they form an interpolation stencil. Master donor is a cell closest to the acceptor cell. The interpolation stencil depends on the chosen overset interpolation scheme.

Each acceptor has a unique master donor, while one donor can be paired

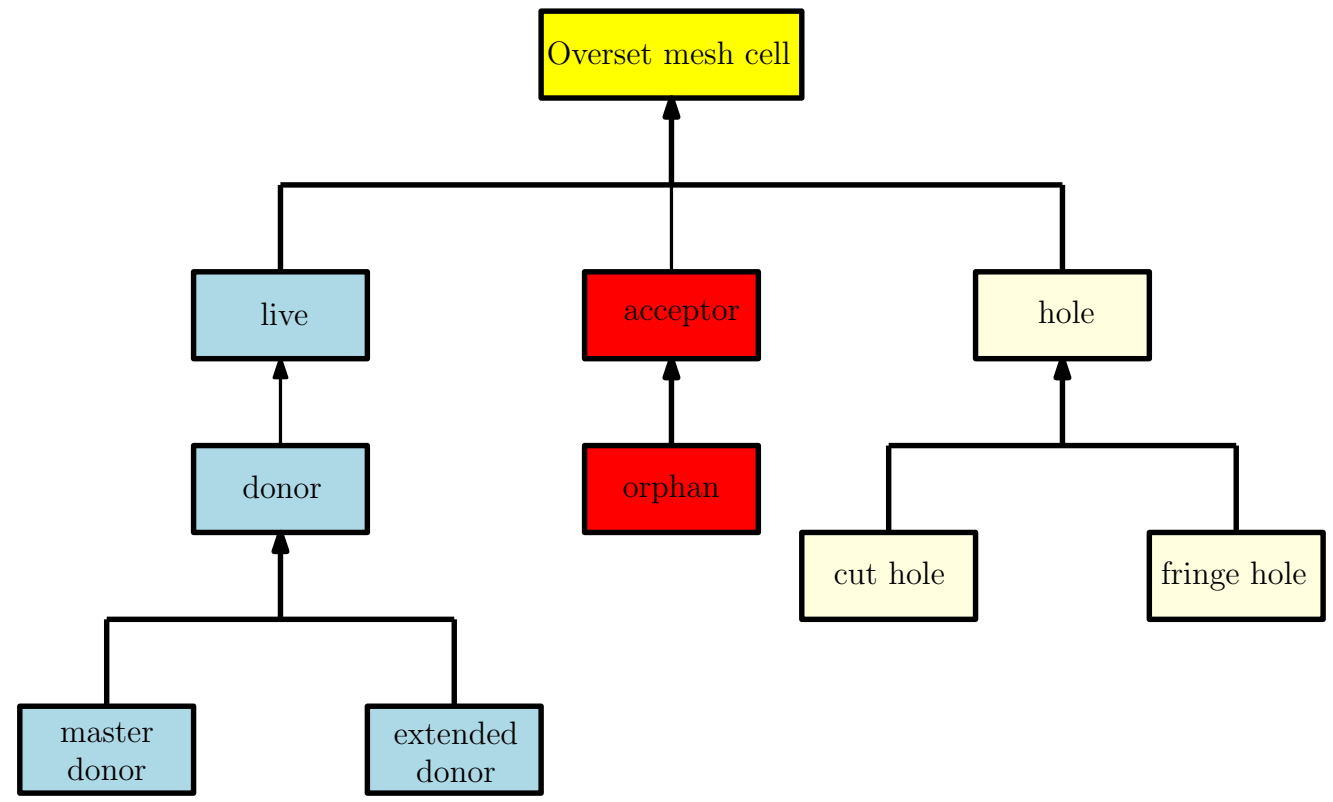

Figure 4: Overset mesh cell types. 
with multiple different acceptors. Pairing one donor with a number of acceptors may lead to an unphysical overall solution, which occurs when the fringe layer of the overset meshes does not match in resolution. Therefore, in the overlapping zone, cells should be of comparable size in both meshes. Also, the coarser of the two meshes determines the level of the discretisation (interpolation) error.

An orphan is an acceptor cell for which donor is found, but its cell centre does not fall within acceptors bounding box. The orphan cell is also called invalid donor/acceptor pair. The presence of an orphan cells generally indicates that there is insufficient overlap between meshes or that the mesh resolutions in the overlapping region do not match well.

If the overset body meshes overlap each other, it is necessary to deactivate all regions on these meshes which are outside of the computational domain, i.e. covered by other bodies or lying outside the background mesh [24]. This process is called hole cutting, and deactivated cells are called cut holes. In addition, since the parts of each mesh may be covered by another overset mesh, the holes may be created in these meshes as well [24].

During the iterative procedure, acceptors that together with donors form unsuitable donor/acceptor pairs are converted to holes in the next iteration. Hole cells which are the result of fringe assembly procedure are called fringe holes.

Also, it is possible that holes or other acceptors surround a specific acceptor cell completely, so this cell may become a hole as well in order to lower possibly time-consuming interpolation. For that purpose, the filtering procedure is employed. The filtering procedure is the final step of the fringe assembly procedure.

The field value in a hole cell does not depend on the field values in neighbouring cells, nor does the hole cell value affect the field values at the neighbouring cells. Field values at hole cells are user-prescribed, i.e. are defined via overset boundary conditions.

\subsection{Fringe assembly algorithm}

The algorithm uses a fringe quality gradient to choose optimal donor/acceptor assembly. Four parameters control the algorithm and all of them have default values. The parameters together with their data types and default values are presented in Table 1. The scalar data type is equivalent to standard C++'s double, label to int and Switch to bool. Among the parameters, the 
user should choose the Donor Suitability Function (DSF) function which calculates the suitability of the donor/acceptor pair indicating their similarity regarding user-prescribed property, usually cell volume.

The presence of orphans is undesirable because if donor and acceptor, which form a single donor/acceptor pair, are far apart, an unphysical solution can be obtained. The orphan suitability is therefore not calculated in a standard manner using DSF, rather it is user prescribed. By prescribing negative suitability to an orphan, average suitability is being degraded. That reduces the possibility that fringe assembly with an orphan present is being chosen for the final overset assembly. By default, orphan suitability is $-100 \%$ which means that the chosen donor is not suitable at all.

The first step in fringe assembly procedure is to get an initial guess for holes and acceptors. The neighbourhood search starts from hole neighbouring cells and optionally from user-specified patches and holes.

After an initial guess is obtained, the algorithm loops through the received donor/acceptor pairs, checks if an orphan is present and if it is, prescribes user-defined suitability. Otherwise, it calculates donor/acceptor suitability using DSF.

Pairs for which suitability is lower than minimum local suitability are categorised as unsuitable while pairs for which suitability is higher than minimum local suitability are categorised as suitable. After passing through all donor/acceptor pairs, the average suitability is calculated.

The algorithm tracks iteration history, i.e. it stores information that is needed to reconstruct fringe layer assembly from a specific iteration and to calculate the slope using the linear regression.

After a user-defined number of iterations is performed and if user prescribed that additional iterations should be done, algorithm performs a linear regression to calculate the suitability slope coefficient, i.e. quality or suitability gradient. Here, average suitability is the predicted variable (on $y$ axis)

Table 1: Algorithm parameters.

\begin{tabular}{|l|c|c|}
\hline Parameter: & Data type: & Default value: \\
\hline minLocalSuit & scalar & 0 \\
\hline specifiedIterationsNumber & label & 4 \\
\hline additionalIterations & Switch & true \\
\hline orphanSuitability & scalar & -1 \\
\hline
\end{tabular}


and the iteration number is the predictor variable ( $x$ axis).

It is assumed that if the gradient is positive, fringe assembly with greater average suitability may be found and an additional iteration will be made. Data stored during the first iteration is then being deleted.

In Fig. 5, as an example, the iterative procedure is demonstrated where the average suitability against the iteration number diagram is shown. After four iterations, the suitability gradient is calculated. Due to a positive suitability gradient, the data from iteration 1 is then deleted. One additional iteration is then made, i.e. iteration 5. After the iteration 5, due to a negative suitability gradient, the iterative procedure is terminated. For final overset fringe assembly, the iteration with the highest average suitability value is chosen, i.e. iteration 4.

The algorithm checks two conditions on each iteration: 1) If there is any unsuitable donor/acceptor pair and 2) If a desired number of iterations is made (i.e. global criterion is satisfied). If those two criteria are not reached, it finds a new set of acceptors. The new acceptors are immediate neighbours of unsuitable acceptors which are eligible (are not holes nor acceptors from current iteration). Acceptors from unsuitable donor/acceptor pairs are converted to holes. Otherwise, the iterative procedure is finished.

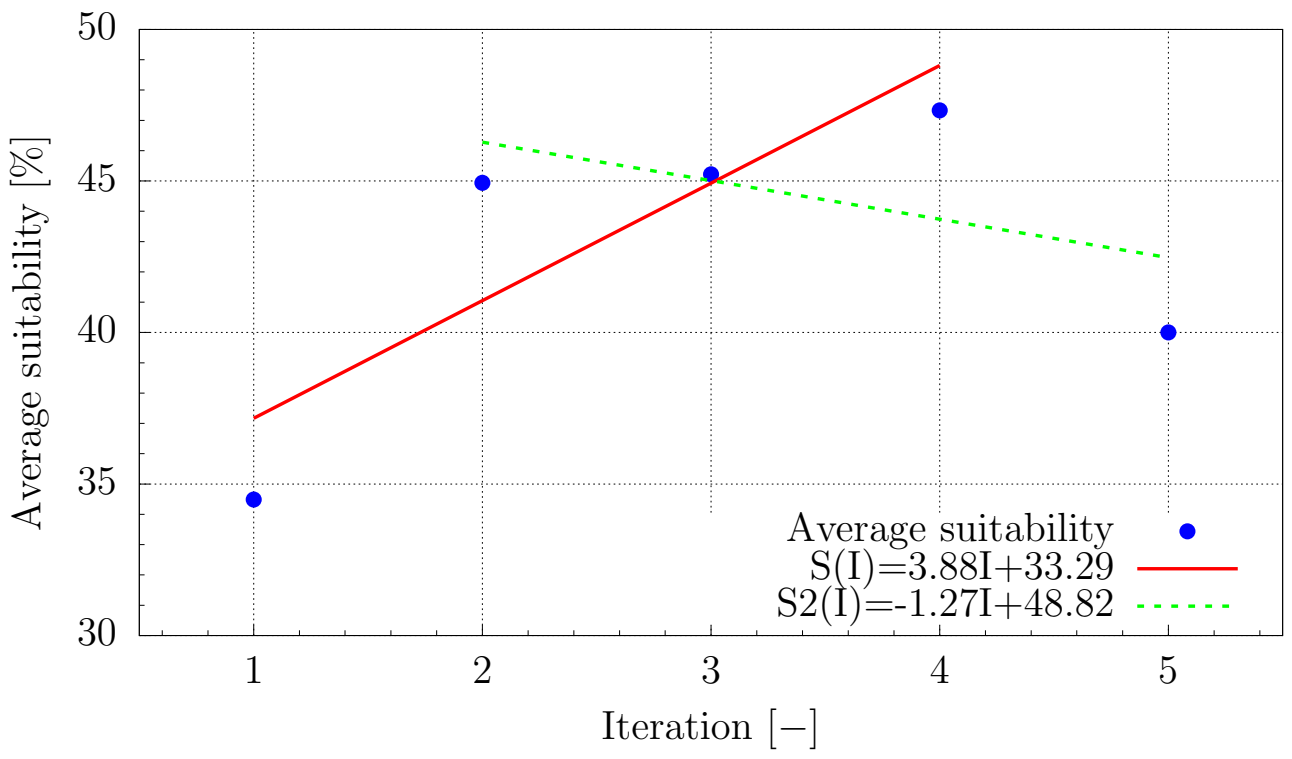

Figure 5: Iterative procedure diagram. 
When the iterative process is finished, the algorithm identifies an iteration with the highest average suitability and performs the pairs filtering procedure: it is possible that holes or other acceptors surround a certain acceptor cell completely, so this cell becomes a hole as well to lower the expense of the overset interpolation.

For more details on the fringe assembly algorithm and overset mesh approach as implemented in foam-extend, the reader is referred to Katavić $[26]$.

\section{Model test and numerical simulation of a chemical tanker launch- ing}

The water basin of the Shipyard "Brodosplit" - Split, Croatia, is shown in Fig.6, indicating the tipping table, layout of the water basin with the water depth. The proposed launching system consists of 13 circular slipways, having radius of $18 \mathrm{~m}$, and distributed equidistantly each $22 \mathrm{~m}$.

The side launching of large ships with trim from the horizontal berth by tipping table is problematic because the ship at erection is supported only by a couple of cradles due to yaw. Thus, the cradle reactions cause high stress concentration on the ship structure, which has to be checked. Also, a large ship launched in such a way in shallow water may touch the bottom, while a high generated wave in a limited water basin may cause other issues.

In order to investigate the above facts, the model tests of the side launching of a 260000 DWT tanker were performed in the Maritime Research Institute Netherlands (MARIN), [11]. An existing wooden model of scale 1:68 was adapted for that purpose. The ship particulars in launching conditions are presented in Table 2.

The ship model on the tipping table is shown in Fig. 6. Two circular slipways were used. The main particulars of slipways and turning pads with force transducers are shown in Fig. 7, where full-scale dimensions are indicated. The port side bilge is supported by two ship-bound cradles. All slide surfaces are equipped with stainless steel polished strips.

Transverse and vertical forces, $F_{y}$ and $F_{x}$, were measured at both ends of the pads by strain gauge force transducers, Fig. 7. The coordinates of the ship's aft and fore peaks, $x, y, z$, were recorded too. The tests were performed varying the ship displacement and position of the centre of gravity in the longitudinal and vertical direction. The recorded ship motion is shown 


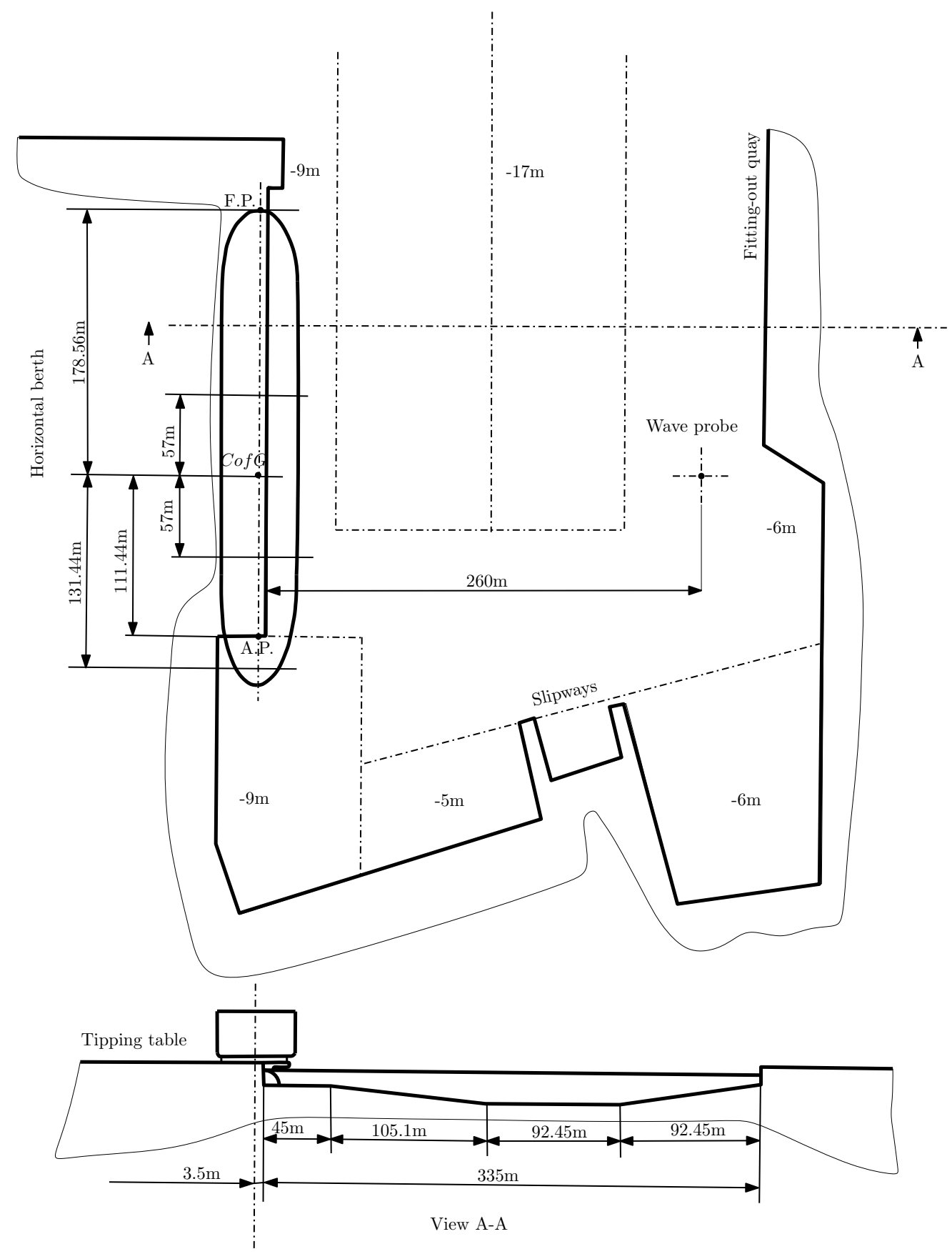

Figure 6: The water basin with ship model on the tipping table. 

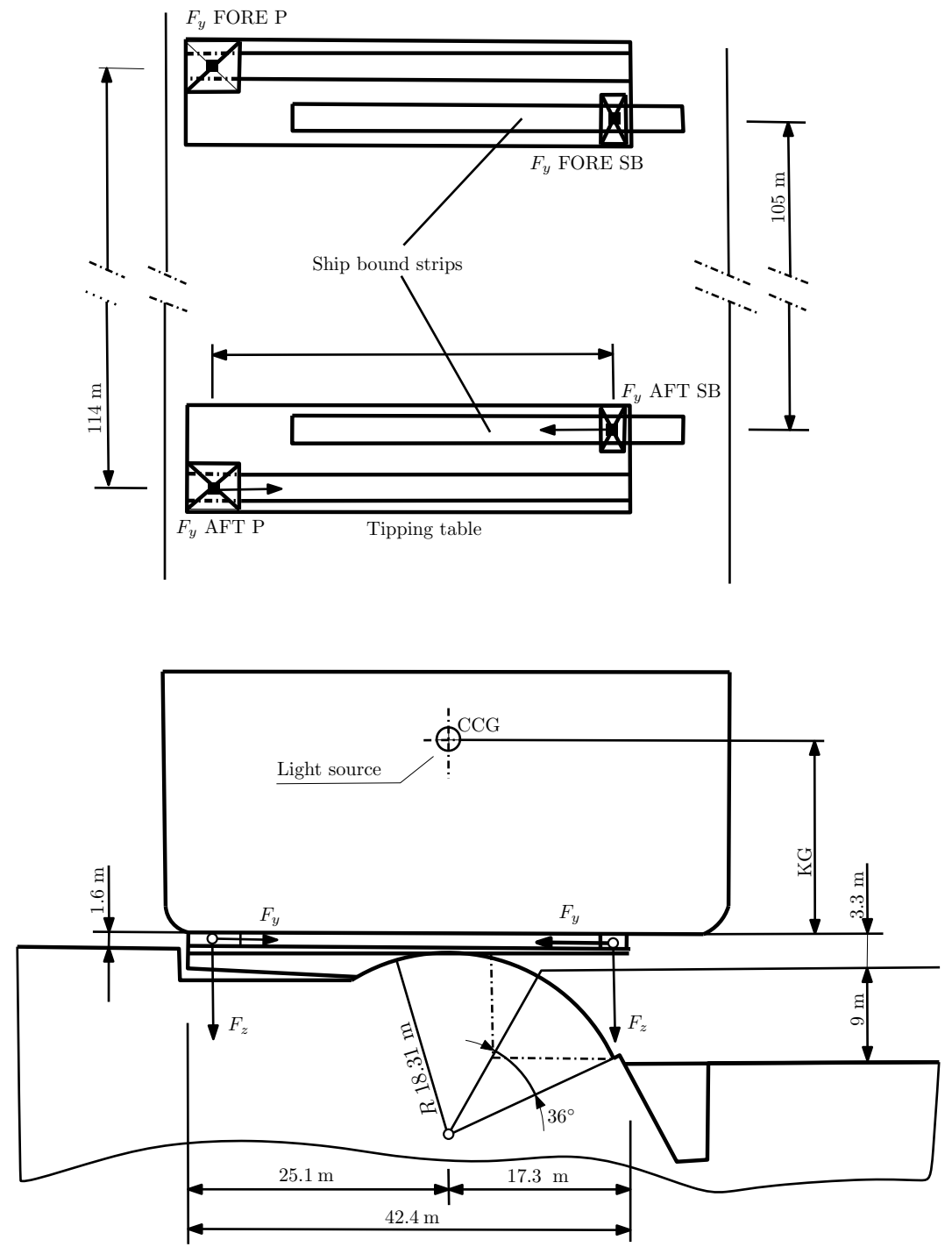

Figure 7: The arrangement of force transducers. 
Table 2: The ship particulars in launching condition.

\begin{tabular}{|l|l|}
\hline Length between perpendiculars & $L_{p p}=310 \mathrm{~m}$ \\
\hline Breadth & $B=53.96 \mathrm{~m}$ \\
\hline Draft fore & $T_{f}=0.45 \mathrm{~m}$ \\
\hline Draft aft & $T_{a}=5.91 \mathrm{~m}$ \\
\hline Displacement & $D=40000 \mathrm{t}$ \\
\hline Centre of gravity above base & $\bar{K} G=18.3 \mathrm{~m}$ \\
\hline Centre of buoyancy from station 0 & $x_{b}=131.4 \mathrm{~m}$ \\
\hline Metacentric height & $G M=56.52 \mathrm{~m}$ \\
\hline Longitudinal radius of gyration & $k_{y y}=87 \mathrm{~m}$ \\
\hline Transverse radius of gyration & $k_{x x}=22.68 \mathrm{~m}$ \\
\hline
\end{tabular}
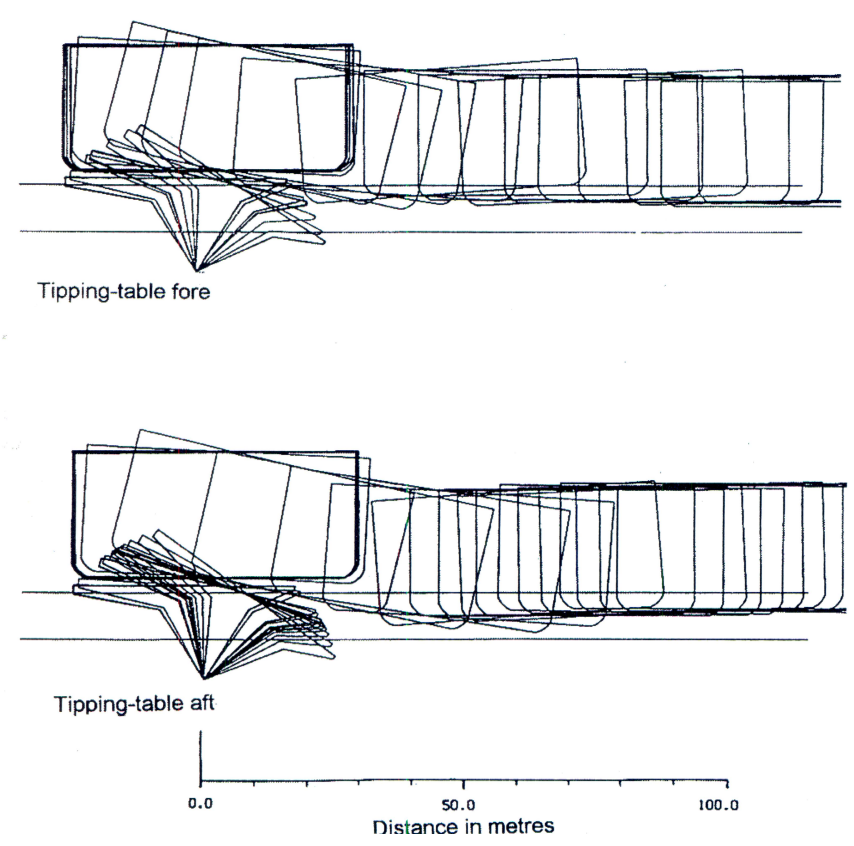

Figure 8: Sketch of the motion of the ship and the pad.

in Fig.8 at the fore and aft pads. Some differences due to yaw motion may be noticed.

Model test and numerical calculation results performed by two pads are elaborated in details in [11] and [12] respectively. Here, the most important results are presented and compared in the time domain. 
The inclination of the ship and the pad are shown in Fig.9. The calculated values for the ship and the pad are related to the cross-section at the longitudinal position of the centre of gravity. The measurement was performed separately for the ship cross-section at the centre of gravity, and the fore and aft pads. The agreement between the calculated and the measured results is relatively good up to the phase 5, when their discrepancies start to increase. The measured inclinations of the ship and the pad are bounded by the calculated values. Thus, the calculation results are on the conservative side.

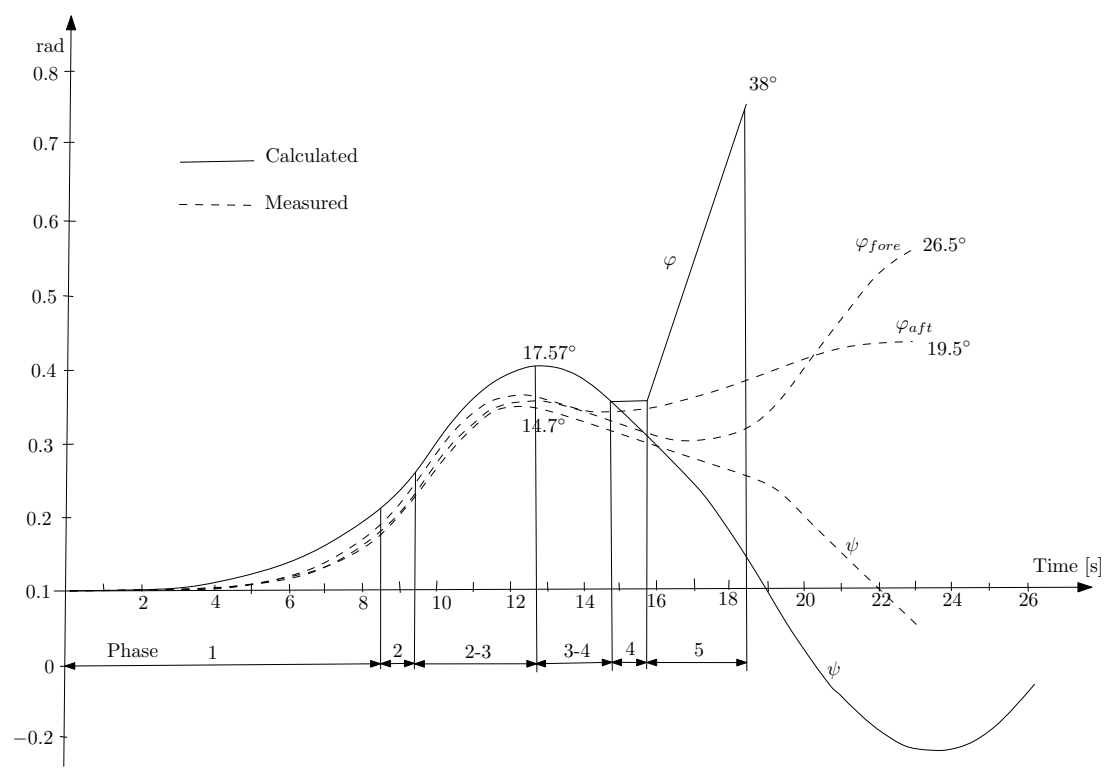

Figure 9: Inclination diagram: $\psi$ - ship angle, $\varphi$ - pad angle.

In [12] additional information on correlation analysis between the calculated and measeured results can be found, i.e. ship yaw and pitch, ship transverse velocity, berth normal reaction and buoyancy as function of time, etc. 


\section{CFD simulation of sideways ship launching}

In this section, the numerical simulation setup and the numerical results are presented in detail. The goal of the simulation is to validate the Naval Hydro Pack [27, 28, 29, 30, 31, 32, 33, 34, 35], combined with the new overset fringe algorithm, on the sideways launching case. The primary validation item is the generated wave amplitude during the sideways launching where the numerical results are compared with the experimental data [11]. According to the experimental data [11], the highest observed wave had a maximum amplitude of $2.2 \mathrm{~m}$, while the lowest maximum amplitude was $1 \mathrm{~m}$. Between those values, a wide scatter of maxima was observed. Measures should be taken for a generated wave of approximately $2 \mathrm{~m}$ [11]. Due to the diffraction effects, the generated wave can reach as much as twice the value observed at the wave probe [11]. Therefore, as a relevant physical quantity for testing and validation, the first generated wave amplitude is considered.

\subsection{Computational domain}

The main data of the water basin with bottom topography and the ship model for the test case No. 5874 from [11] are shown in Fig. 6. According to the coordinate system shown in Fig. 10, the deepest point of the Water Basin is at $-17 \mathrm{~m}$, while the shallowest point is at $-9 \mathrm{~m}$.

A 2D overset mesh is created according to the water basin (see Fig. 6). The computational domain is spatially discretised with two overset meshes. The first mesh is named backgroundMesh and the second one is named shipMesh. The arrangement of the overset meshes and the free surface position at the initial condition are presented in Fig.10.

Dimensions of the backgroundMesh mesh are presented in Fig. 11. The overall mesh length is $396 \mathrm{~m}$, while the overall mesh height is $63 \mathrm{~m}$. Initial

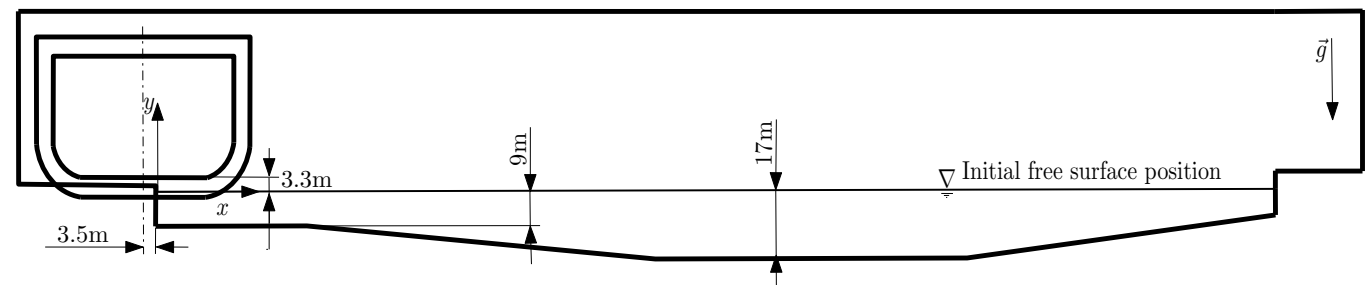

Figure 10: The arrangement of two overset meshes and free surface position at initial condition. 
free surface position is set to $(0,0)$ in accordance with $x-y$ coordinate system (Fig.10).

The shipMesh mesh dimensions are shown in Fig. 12. The overall mesh length is $64 \mathrm{~m}$, while the overall mesh height is $41.05 \mathrm{~m}$.

\subsection{Finite volume overset meshes}

The meshes are block-structured and are generated using the Pointwise software [23]. The backgroundMesh consists of 52724 cells, while the shipMesh mesh consists of 21174 cells. All cells are hexahedral. The overset mesh system thus consists of 73898 cells. To capture the wave properly, the backgroundMesh mesh is made with 20 cells per expected wave height. Also, to ensure a good quality overlap assembly, the shipMesh is generated in a way that the bottom and side blocks consist of 40 layers of cells, while upper blocks, which are not relevant for wave elevation, consist of 18 layers of cells. The combined meshes at the initial condition of side launching are presented in Fig.13.

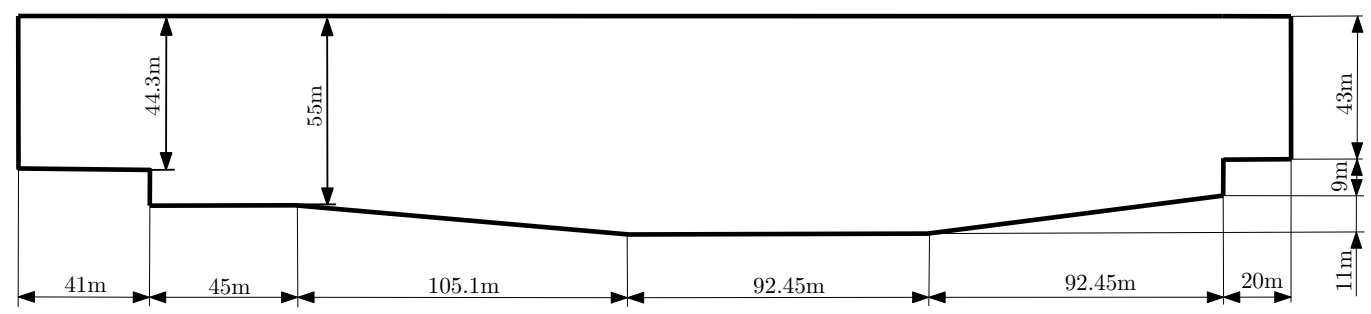

Figure 11: Dimensions of the backgroundMesh region.

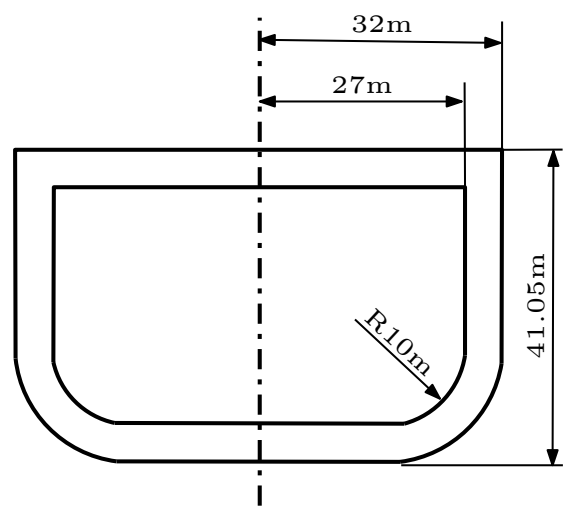

Figure 12: Dimensions of the shipMesh region. 
The donor region for the shipMesh is the backgroundMesh, and vice versa. For overset mesh assembly the adaptive0verlap algorithm presented in Subsection 3.2 with default parameters is employed. The boundaries that determine holes are presented in Table 4.

\subsection{Boundary Conditions}

All computational boundaries are marked in Fig. 14. Prescribed boundary conditions for velocity, phase fraction and dynamic pressure fields are given in Table 3. In addition to the boundary conditions given in Table 3 , overset boundary conditions for every field are presented. The overset boundary conditions are used for overset interpolation, and to prescribe hole cell values for certain fields.

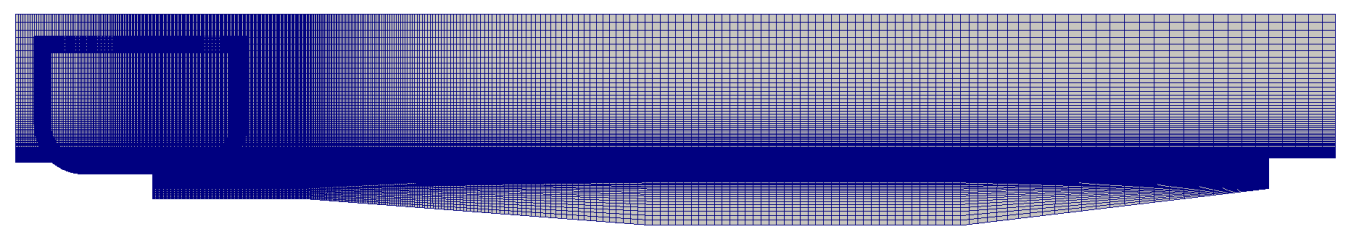

Figure 13: The combined meshes at the initial condition of the side launching simulation.

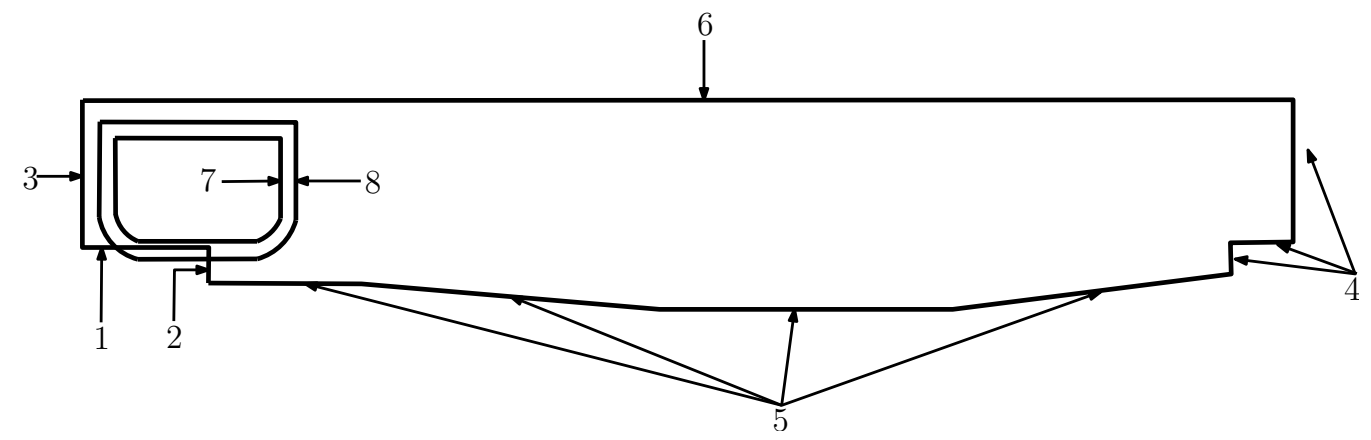

Figure 14: The computational domain with boundary IDs. 
Table 3: Prescribed boundary conditions.

\begin{tabular}{|l|l|l|l|l|}
\hline Patch ID & Patch name & \multicolumn{1}{|c|}{ u } & \multicolumn{1}{c|}{$\alpha$} & \multicolumn{1}{c|}{$p_{d}$} \\
\hline 1 & coastHorizonal & fixedValue & zeroGradient & zeroGradient \\
\hline 2 & coastVertical & fixedValue & zeroGradient & zeroGradient \\
\hline 3 & left & inletOutlet & inletOutlet & zeroGradient \\
\hline 4 & right & fixedValue & zeroGradient & zeroGradient \\
\hline 5 & seabed & fixedValue & zeroGradient & zeroGradient \\
\hline 6 & top & pressureInletOutletVelocity & inletOutlet & fixedValue \\
\hline 7 & boat & movingWallVelocity & zeroGradient & zeroGradient \\
\hline 8 & boatOuter & emptyOverset & emptyOverset & emptyOverset \\
\hline
\end{tabular}

For a general variable $\phi$, following keywords are used for certain boundary conditions:

- Zero gradient (zeroGradient): $\mathbf{n} \bullet \nabla \phi=0$

- Fixed value (fixedValue): $\phi=\phi_{b}$.

The other boundary conditions are explained hereafter:

- Inlet outlet (inletOutlet): the same as zeroGradient, but it switches to fixedValue if the velocity vector next to the boundary is directed towards the inside of the domain (backward flow),

- Pressure inlet outlet velocity (pressureInletOutletVelocity): the same as zeroGradient, but in the case of the backward flow assigns a velocity based on the flux in the patch-normal direction,

- Moving wall velocity (movingWallVelocity): sets the velocity to the desired value for moving walls when employed in moving mesh cases,

- Empty overset (emptyOverset): boundary condition used for overset interpolation and to prescribe hole cell values for certain fields. 
Table 4: Hole-defining boundaries.

\begin{tabular}{|l|l|}
\hline Boundary ID & Name \\
\hline 1 & coastHorizonal \\
\hline 2 & coastVertical \\
\hline 4 & left \\
\hline 5 & right \\
\hline 6 & seabed \\
\hline 7 & top \\
\hline 8 & boat \\
\hline
\end{tabular}

\subsection{Prescribed motions}

The numerical simulations are performed with both the theoretical and the experimental motions prescribed, and wave elevations are compared with the model test result. The experimental data is extracted from diagrams given in [11]. The translational motions are measured for aft and fore of the ship. As 2D computations are performed, the motions are reduced to the centre of gravity of the ship ( $\mathrm{CoG}$ ) using inverse distance weighting (IDW).

The extracted and interpolated motions are shown in Figs. 15 and 16. The extracted motions are denoted with the solid line (fore motion) and the long-dashed line (aft motion), while the interpolated, i.e. prescribed motions are denoted with the short-dashed line. 


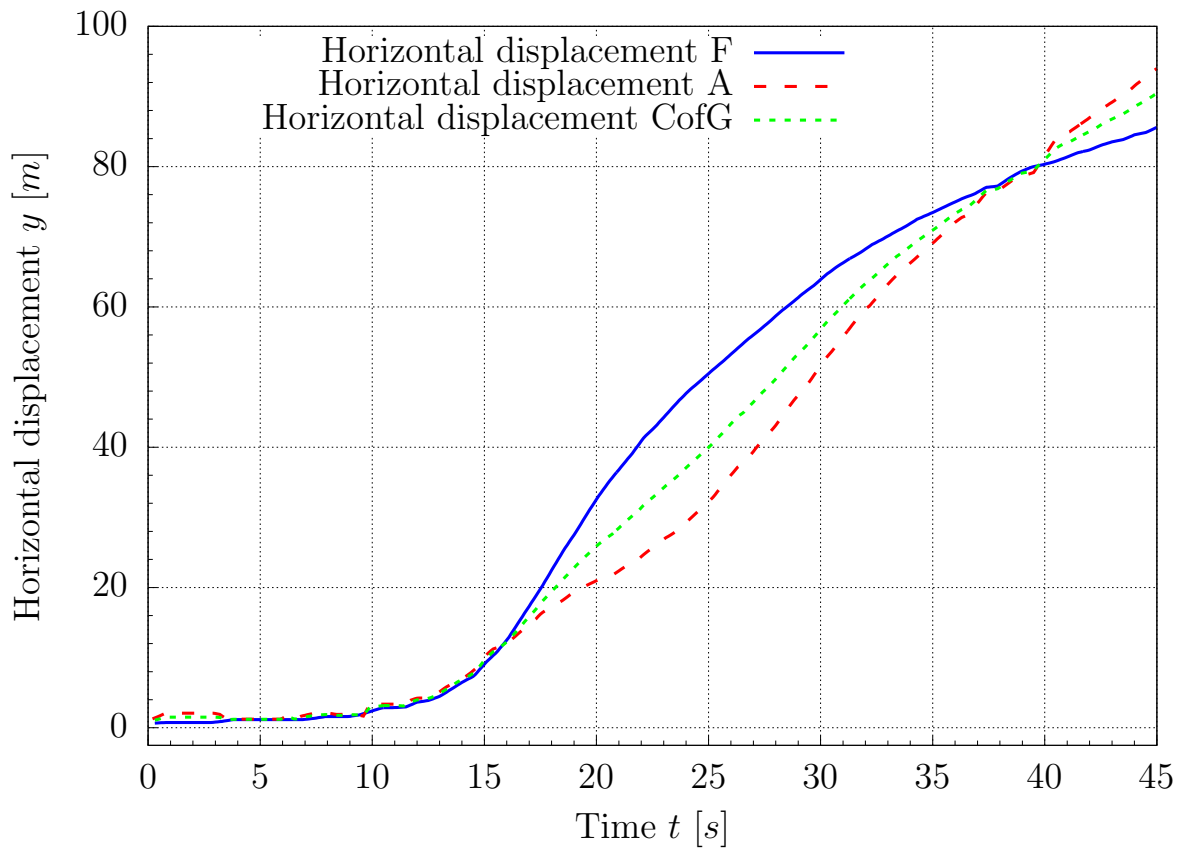

Figure 15: Horizontal displacement (experimental data).

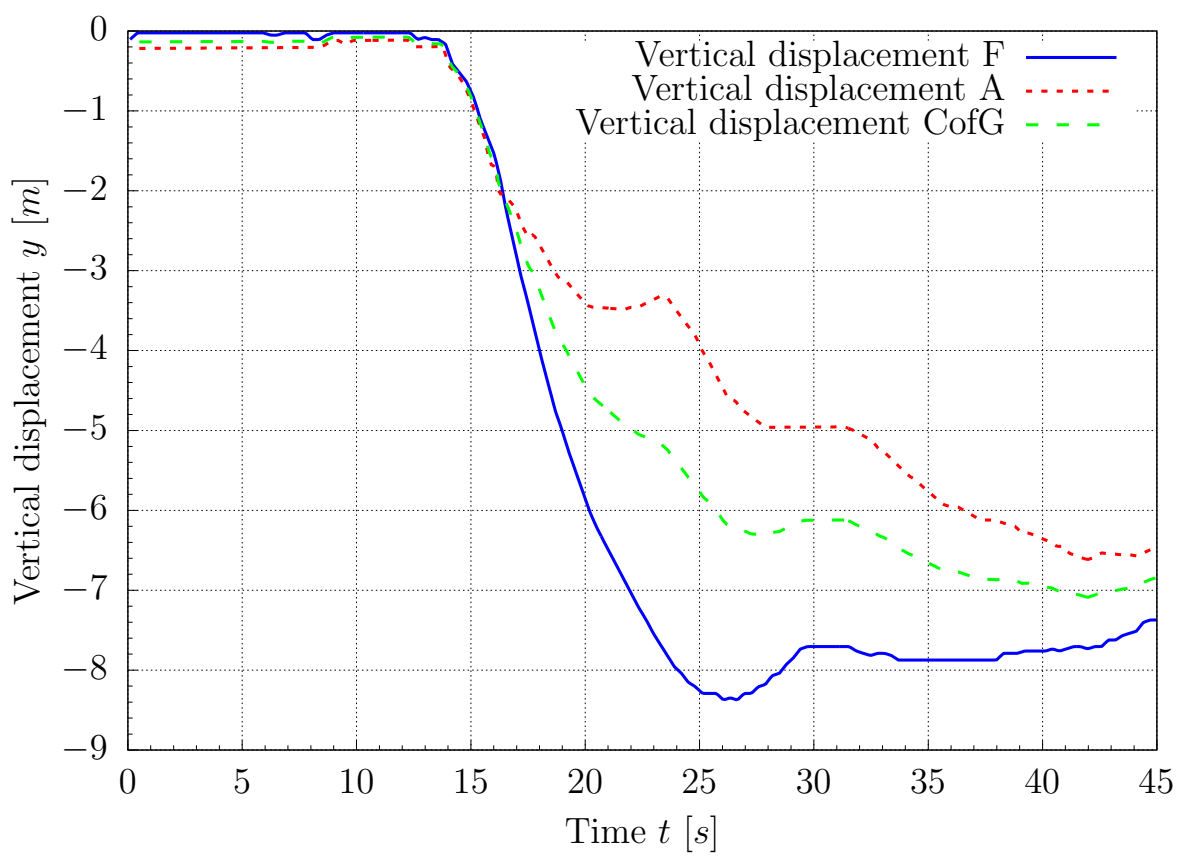

Figure 16: Vertical displacement (experimental data). 


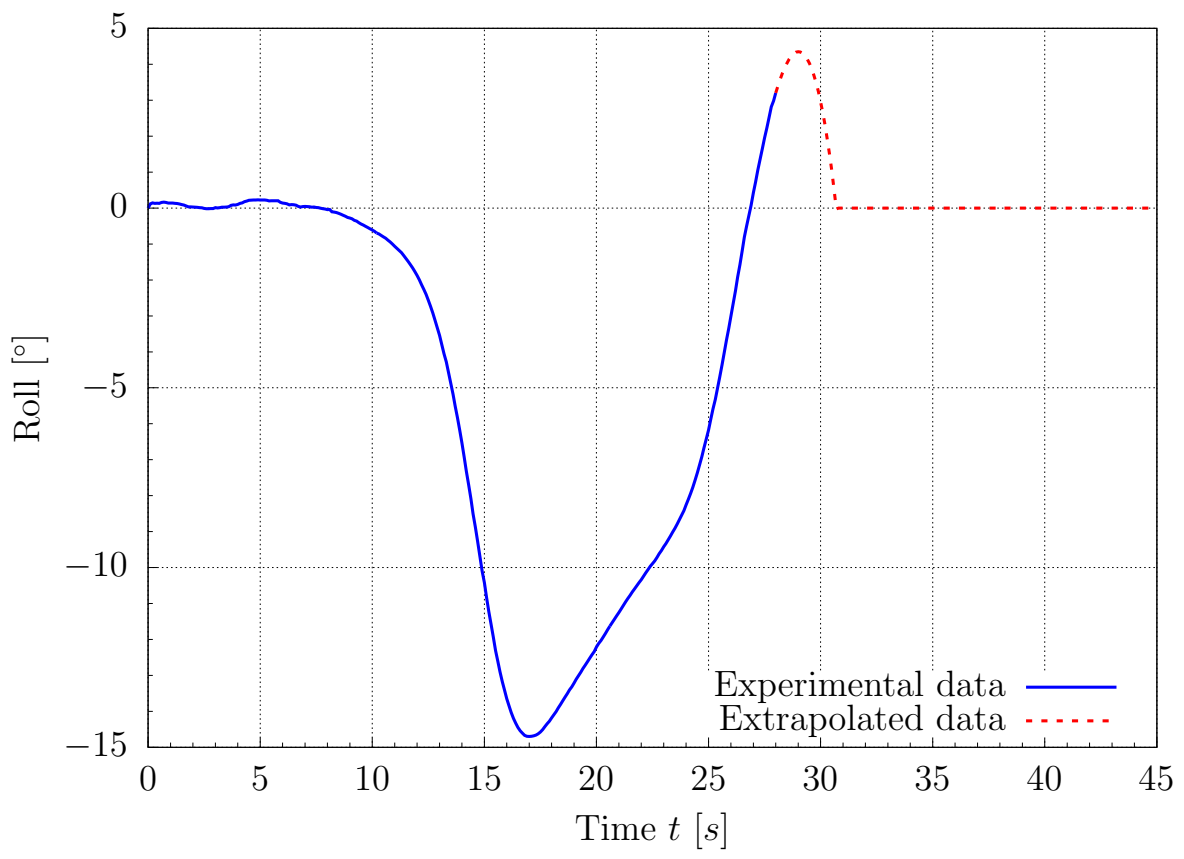

Figure 17: Roll angle (experimental data).

The roll angle is measured only for the first $28 \mathrm{~s}$ and is extrapolated to capture the first wave at the wave probe location. The roll angle is extrapolated in such a way that the ship returns to a neutral position and stays therein, Fig.17.

In Figs. 18 and 19 prescribed theoretical motions are shown. The theoretical motions are obtained by solving equations of motion presented in [7]. Nonlinear differential equations of motion are solved by the finite difference method using the software named LATUP (LAunching by TUrning Pads) [36].

Theoretical motions are available for the first 5 launching phases, i.e. until the final phase, where the ship freely floats performing complex motion. To capture the first wave elevation, motions are extrapolated. 


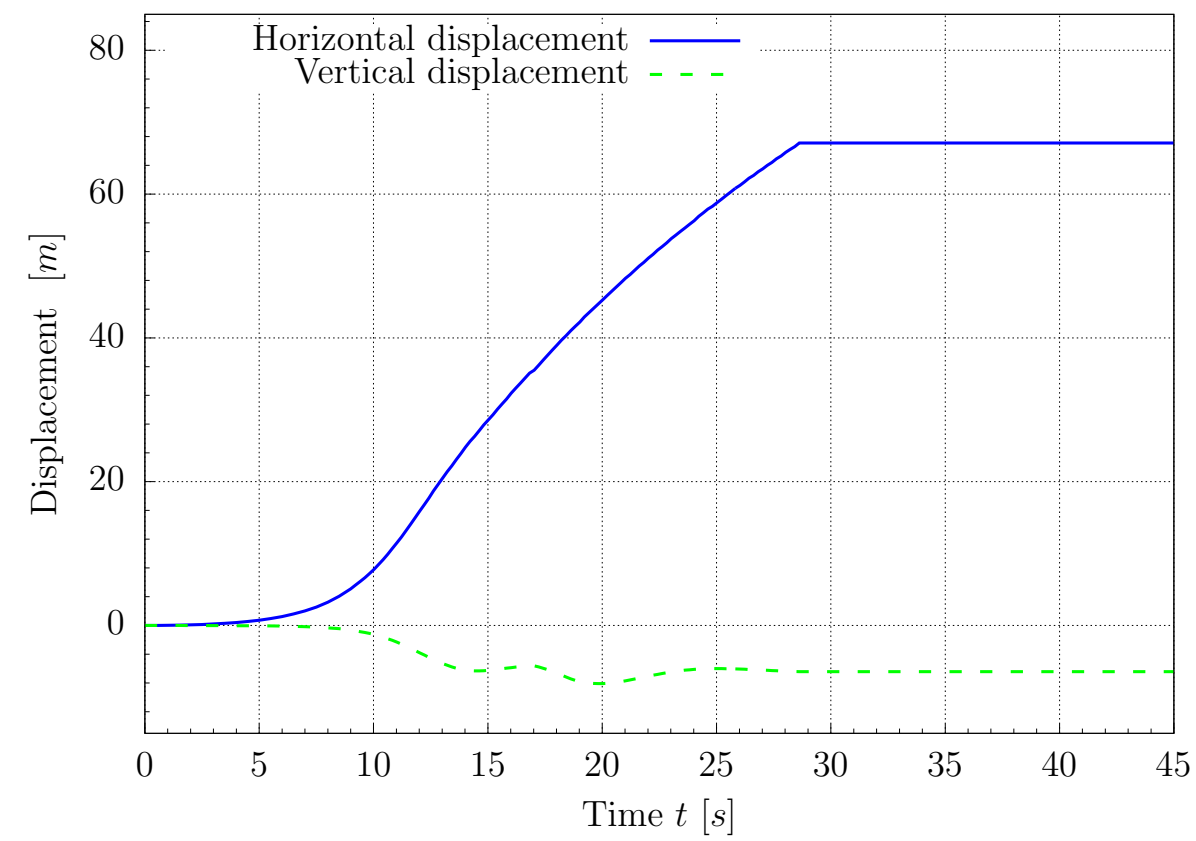

Figure 18: Prescribed horizontal and vertical displacement.

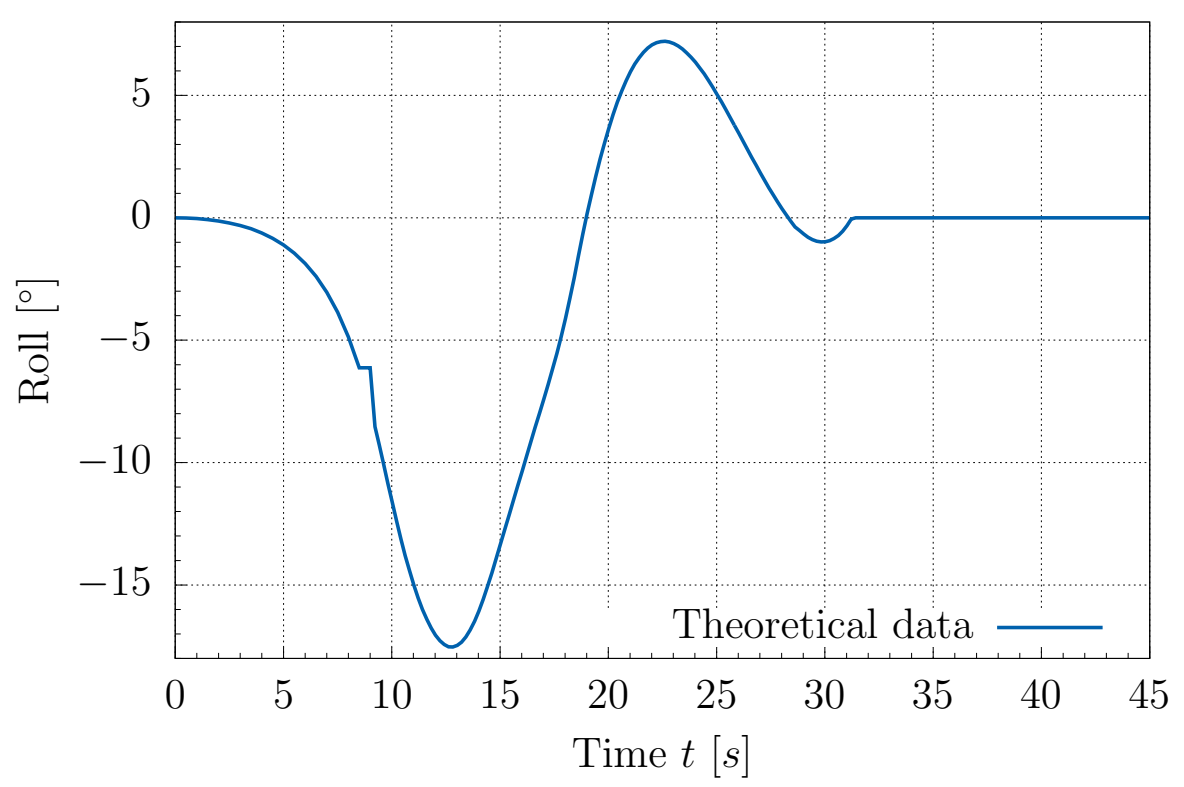

Figure 19: Prescribed roll motion. 


\subsection{Simulation properties}

The density of the water is $\rho_{w}=998 \mathrm{~kg} / \mathrm{m}^{3}$, while the kinematic viscosity is $\nu_{w}=1.05 \cdot 10^{-6} \mathrm{~m}^{2} / \mathrm{s}$. The density of the air is $\rho_{a}=1 \mathrm{~kg} / \mathrm{m}^{3}$, while the kinematic viscosity is $1.48 \cdot 10^{-5} \mathrm{~m}^{2} / \mathrm{s}$. The surface tension coefficient is set to zero because surface tension effects are considered negligible for large-scale flows [37]. No turbulence modelling has been used in this simulation.

At the initial condition the water in the basin is calm and waves are generated exclusively due to ship's motion in water and diffraction effects of the walls and the bottom.

\subsection{Results and discussion}

In Fig. 20 the ship and the water are shown, where the darker shading denotes deeper locations.

As reported in [11], it was observed that the reflected wave flooded the quay from which the ship was launched. In [11], the time of the event is not reported, while in CFD simulation it occurs at $t \geq 69.5 \mathrm{~s}$ (see Fig. 20).

The surface elevation at the probe is given in Fig. 21, where CFD - experimental motions stands for calculated surface elevation using CFD with prescribed experimentally measured motions, EFD stands for the experimentally measured surface elevation, and CFD - theoretical motions stands for the calculated surface elevation using CFD with prescribed theoretical motions.

The maximum measured wave amplitude is $1.997 \mathrm{~m}$, and it is registered at $t=36.04 \mathrm{~s}$, which corresponds to the wave amplitude from experimental test case No. 5874. The maximum calculated wave amplitude (with CFD using the prescribed theoretical motions) is $2.004 \mathrm{~m}$ at $t=37.22 \mathrm{~s}$. The relative discrepancy for the wave amplitude is $0.36 \%$. The phase difference of $1.17 \mathrm{~s}$ between the experimental and the numerical data is larger, with relative discrepancy of $3.16 \%$. The relative discrepancy is calculated as the absolute difference between the measured value and the value obtained by CFD divided by the value of the measured value.

The second wave amplitude is underestimated which is expected because the prescribed roll motion is simply set to zero for $t>28 \mathrm{~s}$ (see Fig. 19).

The difference between the CFD results with prescribed experimental motions and the CFD results with prescribed theoretical motions is noticeable. It occurs because the theoretical motions overestimate the vertical displacement of the ship, i.e. according to the theoretical motions, the ship penetrates deeper into the water than it is the case in experiments. 


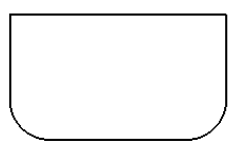

$$
t=0 \mathrm{~s},
$$

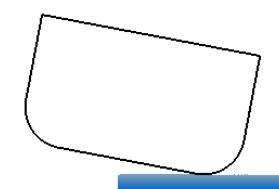

$$
t=15 \mathrm{~s},
$$

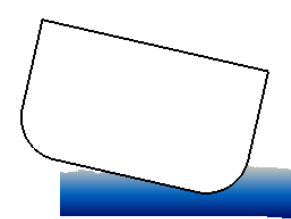

$$
t=19 \mathrm{~s},
$$

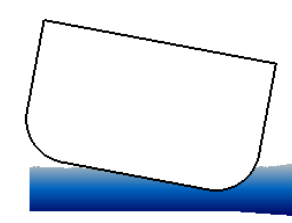

$$
t=21.3 \mathrm{~s} .
$$

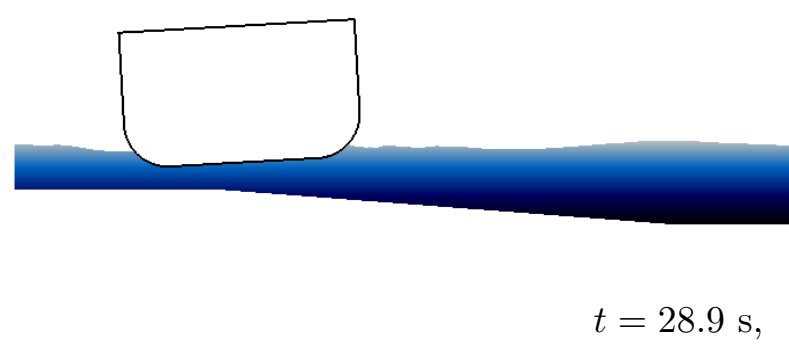

Figure 20: Time-series of the ship launching simulation. 


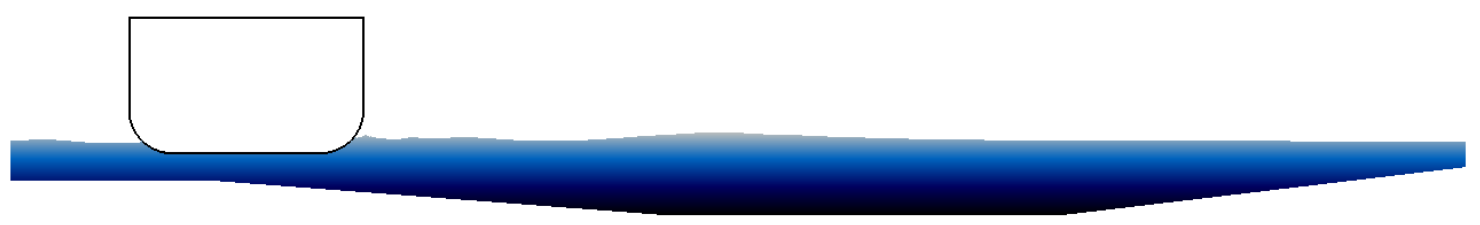

Time, $t=30 \mathrm{~s}$,

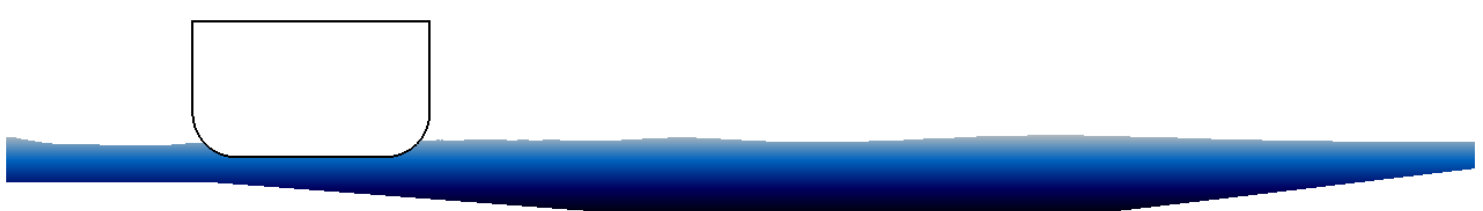

Time, $t=36 \mathrm{~s}$.

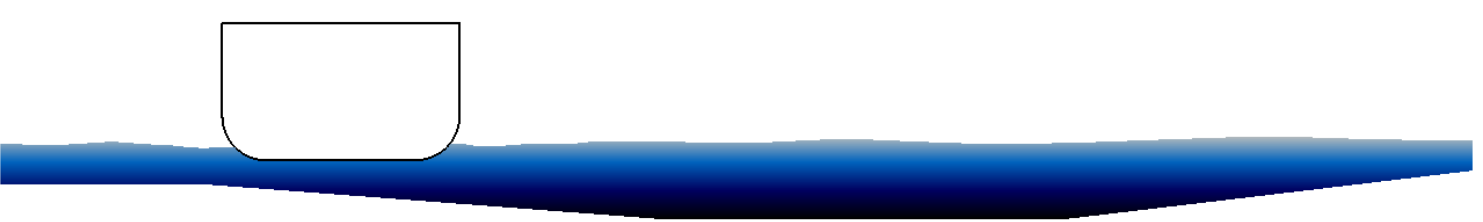

Time, $t=40 \mathrm{~s}$.

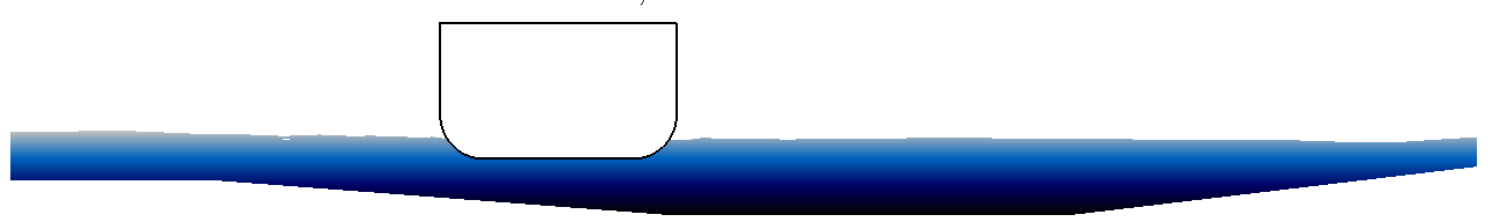

Time, $t=69.5 \mathrm{~s}$.

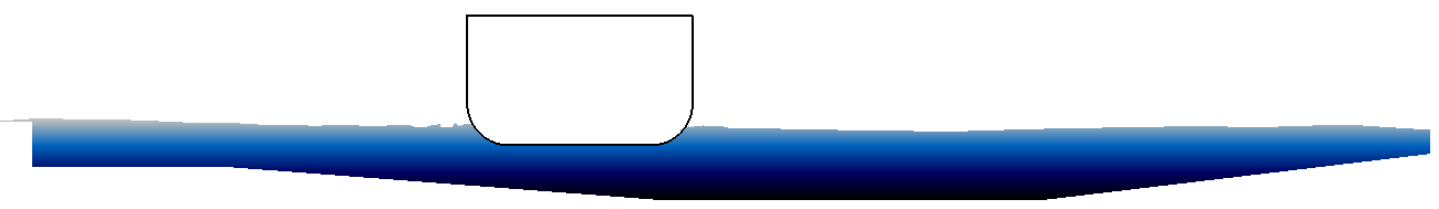

Time, $t=73.5 \mathrm{~s}$.

Figure 20: Time-series of theship launching simulation, continued. 


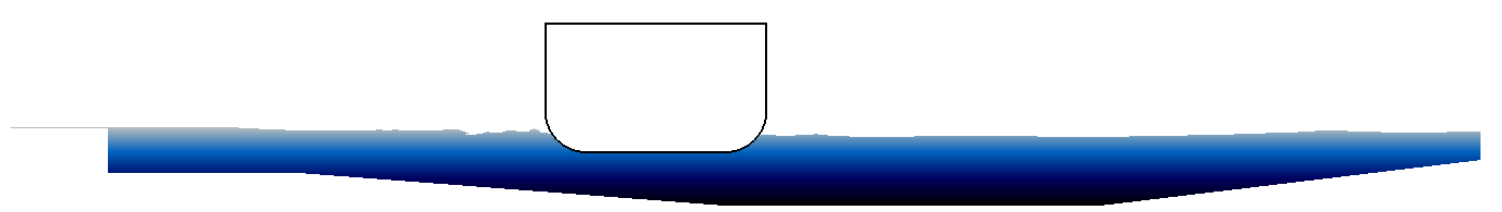

Time, $t=75 \mathrm{~s}$.

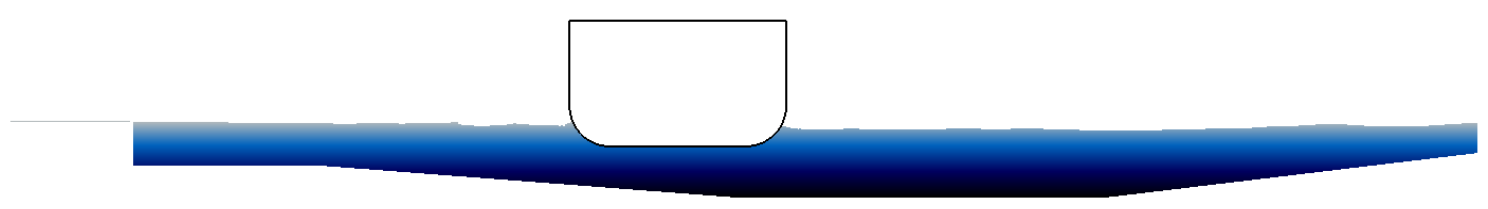

Time, $t=76 \mathrm{~s}$.

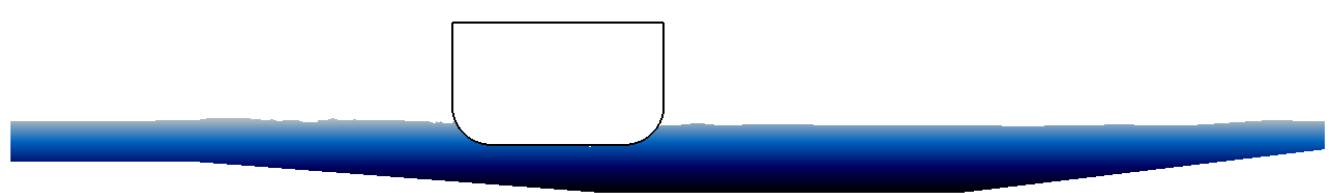

Time, $t=78 \mathrm{~s}$.

Figure 20: Time-series of the ship launching simulation, continued.

When the ship penetrates the water, the mean free surface level is raised for about $0.5 \mathrm{~m}$ due to the $2 \mathrm{D}$ effects. The disturbance propagates after the first wave amplitude is captured.

This indicates that the 2D simulation is an appropriate tool only for capturing the first wave elevation. Also, the maximum measured yaw angle for the test case No. 5874 is $6.5^{\circ}$, which indicates that the ship performs 3D motion in reality. 3D simulation is not performed, because not all water basin dimensions were available, which would also affect the results due to 3D effects. 


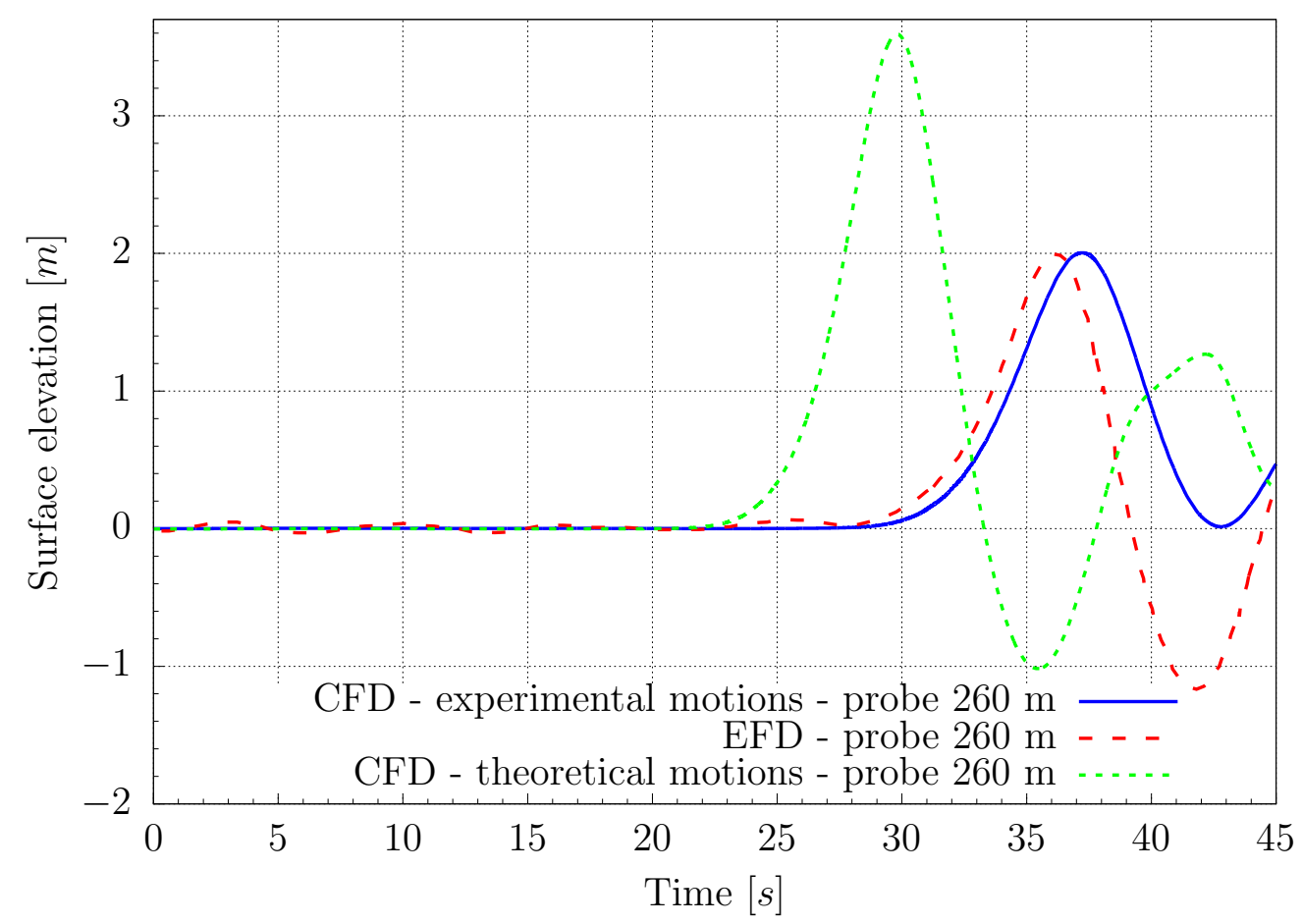

Figure 21: Comparison of the surface elevation.

The numerical calculations were carried out on a desktop computer whose characteristics are presented in Tab. 5. Both of the calculations lasted for 7 hours, therefore less than $30 \mathrm{CPU}$ hours were spent per calculation.

Table 5: Hardware specifications

\begin{tabular}{|l|c|}
\hline CPU & i7-3770@3.4 GHz \\
\hline Number of cores & 4 \\
\hline RAM & 16 GB DDR4 \\
\hline
\end{tabular}

\section{Conclusion}

The extensive numerical and experimental investigation of longitudinal launching of the platform described in [7] and side launching of the tanker show that this idea is feasible also in the case of large floating units. The 
correlation between the calculated and the measured results is acceptable from an engineering point of view, especially in the first part of launching.

Side launching of a ship is more complicated than the longitudinal launching since trim causes yawing during the ship's sliding along the pads. Thus, in phases 4 and 5 the ship is supported only by two cradles, respectively. In order to reduce the load concentration on the ship structure it is necessary to trim the ship close to an even keel.

The main goal of the CFD launching simulation was to compare experimental and numerical results for the first wave amplitude and phase. The relative error between the measured and the calculated wave amplitudes is $0.36 \%$, while the relative error between their phases is $3.16 \%$. It is concluded that the numerical simulation captures side launching physics with high accuracy, thus providing the proof of the validity of 2D CFD approach. More detailed analysis of the wave field would require a 3D CFD simulation.

It is concluded that a $2 \mathrm{D}$ simulation is an appropriate tool only for capturing the first wave elevation, due to 3D effects which occur during side launching of a ship. Furthermore, comparing simulations with prescribed experimental and theoretical motions shows significant sensitivity of the wave amplitude to the imposed motions.

The advantage of launching the ships and offshore structures by tipping table arrangement is the possible introduction of higher level of mechanisation and automation in the ship-building process, simultaneous building of several units employing only one launching system, and cost reduction in comparison with the classical method of ship-building. Sideways ship launching is also performed when a ship is built in a yard on a river or canal either because the hull is especially long or the channel it is being launched into is narrow.

\section{Acknowledgements}

This investigation received funding within the International cooperation project Global Core Research Centre for Ships and Offshore Plants (GCRC SOP Grant No. 2011-0030669), established by the South Korean Government (MSIP) through the National Research Foundation of South Korea (NRF). The authors would like to express their gratitude to Dr. Guilherme Vaz for sharing the original report of the experimental campaign performed in MARIN, Netherlands. 


\section{References}

[1] Vukman, Š.. Tipping launching ways in modern shipbuilding technology (in Croatian). Brodogradnja, 29(5):317-322, 1981.

[2] Vukman, Š.. Launching by means of tipping table arrangement (in Croatian). Brodogradnja, ,30(2):59-68, 1982a.

[3] Vukman, Š.. Construction of ships on horizontal level with launching over tipping table. Brodogradnja,, 30(2):135-141, 1982b.

[4] Vukman, Š., Krstulović, A., and Papić, J. Method of launching by means of polymeric materials instead of lubricants. Brodogradnja,, 34(2):99$102,1986$.

[5] Senjanović, I., Ljubetić, M., and Vukman, Š. Investigation of launching of ships and offshore structures from horizontal berth by tipping table principle. International Shipbuilding Progress, 33(388):218-230, 1986.

[6] Senjanović, I. Launching of ships and floating structures from horizontal berth by tipping table. Journal of Ship Production, 14(4):265-276, 1998b.

[7] Senjanović, I. Launching theory of ships from horizontal berth by tipping table arrangement. Brodogradnja, 46(4):359-369, 1998a.

[8] Senjanović, I. and Grubišić, I. Side launching of ships from horizontal berth by tipping table arrangement. Brodogradnja, 47(2):135-143, 1999.

[9] Dormand, J. R. and Prince, P. J. A family of embedded Runge-Kutta formulae. Journal of computational and applied mathematics, 6(1):1926,1980 .

[10] Anzulović, I. A dynamic model of turning slipway's returning motion. Brodogradnja, 45(1):44-50, 1997.

[11] Feikema, G., Luisman, H., and Pinkster, J. Report No. 010001-1-BT: Launching tests for a 260000 DWT ship. Maritime Research Institute Netherlands (MARIN), 1989. 
[12] Senjanović, I. Numerical and experimental investigation of side launching of tanker by tipping table principle. Brodogradnja, 46(3):264-270, 1998c.

[13] Abdulaj, R. and Ljubetić, M. The effect of eccentricity on kinematics of side launching with rotative standing ways (in Croatian). Proceedings of the 11th symposium Theory and Practice of Ship-building in Memoriam Professor Leopold Sorta, Dubrovnik, 1994.

[14] Čagalj, A. Building a barge on a horizontal berth (in Croatian). Brodogradnja, 43(3):58-62, 1995.

[15] Vukčević, V., Jasak, H., and Gatin, I. Implementation of the ghost fluid method for free surface flows in polyhedral finite volume framework. Computers \& Fluids, 153:1-19, 2017.

[16] Desjardins, O., Moureau, V., and Pitsch, H. An accurate conservative level set/ghost fluid method for simulating turbulent atomization. Journal of Computational Physics, 227(18):8395-8416, 2008.

[17] Huang, J., Carrica, P., and Stern, F. Coupled ghost fluid/two-phase level set method for curvilinear body-fitted grids. International Journal for Numerical Methods in Fluids, 55(9):867-897, 2007.

[18] Wilcox, D. Turbulence modeling for CFD, volume 2. DCW industries La Canada, CA, 1998.

[19] Batchelor, C. K. An introduction to fluid dynamics. Cambridge University Press, 1967.

[20] Ito, K. and Li, Z. Interface conditions for stokes equations with a discontinuous viscosity and surface sources. Applied mathematics letters, 19(3):229-234, 2006.

[21] Ubbink, O. and Issa, R. A method for capturing sharp fluid interfaces on arbitrary meshes. Journal of Computational Physics, 153(1):26-50, 1999.

[22] Rusche, H. Computational fluid dynamics of dispersed two-phase flows at high phase fractions. PhD thesis, Imperial College London (University of London), 2003. 
[23] Wyman, N. Why You Should Be Using Overset Grids. http://www.pointwise.com/theconnector/2014-September/ Why-You-Should-Be-Using-Overset-Grids.htm, 2014. [Online, accessed 5 June 2019].

[24] Hadzic, H. Development and application of finite volume method for the computation of flows around moving bodies on unstructured, overlapping grids. Technische Universität Hamburg, 2006.

[25] Celeritas Simulation Technology, LLC. What is Overset? http:// celeritassimtech. com, 2019. [Online, accessed 5 June 2019].

[26] Katavić, J. Development of automatic fringe assembly algorithm for overset meshes in foam-extend software. Master's thesis, Faculty of Mechanical Engineering and Naval Architecture, University of Zagreb, Croatia, 2018.

[27] Jasak, H. CFD analysis in subsea and marine technology. In IOP Conference Series: Materials Science and Engineering, volume 276, page 012009. IOP Publishing, 2017.

[28] Jasak, H., Vukčević, V., Gatin, I., and Lalović, I. Cfd validation and grid sensitivity studies of full scale ship self propulsion. International Journal of Naval Architecture and Ocean Engineering, 11(1):33-43, 2019.

[29] Gatin, I., Cvijetić, G., Vukčević, V., Jasak, H., and Malenica, Š. Harmonic balance method for nonlinear and viscous free surface flows. Ocean Engineering, 157:164-179, 2018.

[30] Gatin, I., Vukčević, V., Jasak, H., and Rusche, H. Enhanced coupling of solid body motion and fluid flow in finite volume framework. Ocean Engineering, 143:295-304, 2017.

[31] Gatin, I., Vukčević, V., and Jasak, H. A framework for efficient irregular wave simulations using higher order spectral method coupled with viscous two phase model. Journal of Ocean Engineering and Science, 2(4):253-267, 2017.

[32] Gatin, I., Vukčević, V., Jasak, H., Seo, J., and Rhee, S. CFD verification and validation of green sea loads. Ocean Engineering, 148:500-515, 2018. 
[33] Jasak, H., Gatin, I., and Vukčević, V. Monolithic coupling of the pressure and rigid body motion equations in computational marine hydrodynamics. Journal of Marine Science and Application, 16(4):375-381, 2017.

[34] Vukčević, V., Jasak, H., and Malenica, Š. Decomposition model for naval hydrodynamic applications, part I: Computational method. Ocean Engineering, 121:37-46, 2016.

[35] Vukčević, V., Jasak, H., and Malenica, Š. Decomposition model for naval hydrodynamic applications, part II: Verification and validation. Ocean Engineering, 121:76-88, 2016.

[36] Senjanović, I. Building of ships and floating structures on horizontal berth and their launching by tipping table principle. In The Proceedings of the International Offshore and Polar Engineering Conference. International Society of Offshore and Polar Engineers, 1999.

[37] Carrica, P. M., Wilson, R. V., Noack, R. W., and Stern, F. Ship motions using single-phase level set with dynamic overset grids. Computers $\&$ Fluids, 36(9):1415-1433, 2007. 